\title{
The Structure of hDmc1 Filaments and Ambiguities in Helical Reconstruction
}

\author{
Xiong Yu and Edward H. Egelman \\ Department of Biochemistry and Molecular Genetics, University of Virginia, Box 800733, \\ Charlottesville, VA 22908-0733
}

Proteins in the $\operatorname{RecA} / \operatorname{Rad} 51 / \operatorname{RadA}$ family form nucleoprotein filaments on DNA that catalyze a strand exchange reaction as part of homologous genetic recombination. Because of the centrality of this system to many aspects of DNA repair, the generation of genetic diversity, and cancer when this system fails or is not properly regulated, these filaments have been the object of many biochemical and biophysical studies. A recent paper has argued that the human Dmc1 (hDmc1) protein, a meiotic homolog of bacterial RecA and human Rad51, forms filaments on single stranded DNA with $\sim 9$ subunits per turn in contrast to the filaments formed on double stranded DNA with $\sim 6.4$ subunits per turn, and that the stoichiometry of DNA binding is different between these two filaments.

We show using scanning transmission electron microscopy (STEM) that both filament forms have similar mass per unit length, which is that expected from $\sim 6.4$ subunits per turn. More generally, we show how ambiguities in helical symmetry determination can generate incorrect solutions, and why one sometimes must use other techniques, such as biochemistry, metal shadowing, or STEM to resolve these ambiguities. While three-dimensional reconstruction of helical filaments from EM images is a powerful tool, the intrinsic ambiguities that may be present are not sufficiently appreciated. 